\title{
Reflets
}

Revue d'intervention sociale et communautaire

\section{L'utilisation des technologies d'information et de communication chez les femmes francophones en situation minoritaire au Canada}

\section{Ann Denis et Michèle Ollivier}

Volume 9, numéro 1, printemps 2003

Le genre en contexte : pratiques sociales et représentations

URI : https://id.erudit.org/iderudit/010870ar

DOI : https://doi.org/10.7202/010870ar

Aller au sommaire du numéro

Éditeur(s)

Reflets : Revue ontaroise d'intervention sociale et communautaire

ISSN

1203-4576 (imprimé)

1712-8498 (numérique)

Découvrir la revue

Citer cet article

Denis, A. \& Ollivier, M. (2003). L'utilisation des technologies d'information et de communication chez les femmes francophones en situation minoritaire au Canada. Reflets, 9(1), 222-230. https://doi.org/10.7202/010870ar

Tous droits réservés (C) Reflets : Revue ontaroise d'intervention sociale et communautaire, 2002
Ce document est protégé par la loi sur le droit d'auteur. L'utilisation des services d'Érudit (y compris la reproduction) est assujettie à sa politique d'utilisation que vous pouvez consulter en ligne.

https://apropos.erudit.org/fr/usagers/politique-dutilisation/ 


\section{L'utilisation des technologies d'information et de}

communication chez les femmes francophones en situation minoritaire au Canada

\section{Ann Denis et Michèle Ollivier}

Université d'Otawa

Au cours des dix dernières années, les questions liées aux technologies d'information et de communication (TIC) ont suscité de nombreux débats. Ces débats s'inscrivent dans un discours plus général sur les sociétés dites du savoir ou de l'information, selon lequel la capacité de produire et d'utiliser les connaissances, surtout celles qui sont de nature scientifique et technique, aurait remplacé l'accès aux matières premières et à l'énergie comme moteur de la croissance économique. On parle aussi de plus en plus de sociétés en réseaux, dans la mesure où les affiliations sociales fluides et contingentes seraient en voie de remplacer les identités collectives plus permanentes ancrées dans un espace physique et une mémoire commune. Parce qu'elles donnent accès à la fois à des sources quasi-illimitées d'information et à des réseaux sociaux qui transcendent les barrières géographiques, les technologies d'information et de communication occupent une place centrale dans ce discours. Elles feraient désormais partie des compétences de base essentielles à la réussite scolaire, à l'exercice d'un emploi et, de façon plus générale, à l'inclusion sociale et à l'exercice de la citoyenneté dans la société du savoir. 
C'est en tenant compte de ces débats que nous avons réalisé, au cours des cinq dernières années, trois recherches sur l'utilisation des technologies d'information et de communication par les francophones en situation minoritaire au Canada. La première portait sur les jeunes de la fin du secondaire (Denis et Ollivier, 2002) dans des écoles francophones de quatre régions de l'Ontario (Ottawa, Est, Toronto et Nord). La seconde, réalisée en collaboration avec la Fédération nationale des femmes canadiennesfrançaises et Industrie Canada, portait sur l'utilisation d'Internet par les groupes de femmes francophones en situation minoritaire au Canada (Ollivier et Denis, 2002). Dans la troisième, nous avons utilisé les données de l'Enquête sociale générale 2000 de Statistique Canada pour analyser les différences dans l'accès et l'usage entre les hommes et les femmes et, en considérant seulement les femmes, selon la langue d'usage à la maison, le revenu et l'éducation (Denis et Ollivier, sous presse). Nous rendons compte ici des deux dernières recherches, qui portent principalement sur les femmes.

\section{L'enquête sociale générale 2000 de Statistique Canada}

Tous les travaux réalisés au cours des dix dernières années sur l'accès aux technologies d'information et de communication et sur les usages qui en sont faits arrivent à des conclusions similaires (voir Fong et al., 2001, pour un survol récent des enquêtes canadiennes et américaines). De façon générale, l'accès à l'ordinateur et à Internet reproduit assez fidèlement les sources habituelles d'inégalités sociales. Au Canada et ailleurs dans le monde, les recherches montrent des différences persistantes, tant en ce qui concerne l'accès à la technologie que l'intensité et la diversité des usages, selon le revenu, l'éducation, l'âge, la composition du ménage (familles biparentales par rapport aux monoparentales), la région (urbaine/rurale), la langue d'usage (anglais/français) et la province de résidence (l'Est étant en général moins branché que le Centre et l'Ouest du pays). Les recherches montrent également que l'écart numérique a considérablement diminué 
au cours des années, notamment entre les femmes et les hommes de même qu'entre francophones et anglophones.

Dans notre analyse des données de l'Enquête sociale générale de Statistique Canada, nous avons cherché, premièrement, à déterminer jusqu'à quel point l'écart numérique persiste entre les sexes de même qu'entre francophones et anglophones et, deuxièmement, à identifier certains des facteurs responsables de ces écarts. Notre analyse indique, d'une part, que les femmes continuent à être moins nombreuses que les hommes à avoir accès à un ordinateur et à Internet à la maison et, d'autre part, qu'un écart similaire persiste entre femmes francophones et anglophones. Quand on examine les différences d'accès à l'ordinateur et à Internet à la maison selon le sexe en tenant compte du revenu du ménage, on constate que les écarts sont presque entièrement attribuables à des facteurs économiques. À revenu égal, les différences entre les sexes disparaissent entièrement, sauf chez les ménages gagnant moins de $20000 \$$ par année, où les hommes sont plus nombreux que les femmes à avoir accès à l'ordinateur et à Internet à la maison. On peut en conclure que si les femmes sont moins branchées que les hommes, c'est en partie parce qu'elles sont plus nombreuses dans les catégories inférieures de revenu. La réduction de l'écart numérique entre les sexes passerait donc dans ce cas par une amélioration de la situation économique des femmes.

Nous avons également examiné les différences entre les femmes francophones (qui parlent principalement le français à la maison) et les femmes anglophones (qui parlent principalement l'anglais). Si on compare les femmes des deux groupes linguistiques à niveau égal de revenu et d'éducation, on constate que les femmes anglophones, à tous les niveaux de revenu, demeurent plus nombreuses que les femmes francophones à avoir accès à Internet à la maison. Ces résultats indiquent que des facteurs liés directement à la langue parlée à la maison continuent d'influencer l'accès aux technologies d'information et de communication. Nous savons peu de choses sur ces facteurs. Dans une étude des groupes de femmes réalisée au Québec par Relais-Femmes, les écarts entre les groupes linguistiques étaient attribués entre autres 
à la faiblesse du contenu francophone sur le Web et à l'absence d'une masse critique de groupes branchés (Duque, 1998).

En ce qui concerne l'intensité (le nombre d'heures passées en ligne) et la diversité (genres d'activités pratiquées) des usages, on constate que les femmes continuent de passer moins de temps en ligne à comparer aux hommes et à pratiquer une gamme moins diversifiée d'activités. On observe des différences similaires entre femmes francophones et femmes anglophones. Selon certaines analyses, la réduction de l'écart numérique concernant l'accès à Internet masquerait d'autres types de divergences plus marquées. Ainsi, pour Manuel Castells, on assisterait actuellement à une polarisation entre usagers interagisssants (interacting), qui utilisent les nouveaux médias à leur plein potentiel, et les interagis (interacted), qui doivent se contenter d'un ensemble plus limité d'applications et de choix pré-conditionnés (Castels, 1996).

Dans nos travaux, nous remettons en question l'utilité de cette division binaire du monde entre classes ou groupes interagissants (actifs, créatifs et dominants) et interagis (passifs et dominés). En effet, de nombreuses féministes ont dénoncé les effets pervers des oppositions binaires qui présentent uniformément certains groupes (les femmes en général, les femmes du Tiers Monde, les pauvres, les immigrantes) comme victimes passives de systèmes économiques et patriarcaux tandis que d'autres groupes (les hommes, les femmes de classe moyenne, les femmes des pays développés) sont invariablement présentés comme éduqués, avancés et agissants (voir entre autres Mohanty, 1997). Ces critiques rejoignent celles qui dénoncent le "modèle déficitaire " du rapport des femmes à la technologie, selon lequel le moindre nombre d'heures passées en ligne par les femmes serait une indication d'un "retard " qu'il faudrait combler par des politiques incitatives. Ce type d'analyse tend à prendre le comportement masculin (intensité de l'usage) comme la norme et les comportements féminins comme des "problèmes » auxquels il faut apporter des «solutions".

Pourtant, on peut se demander si le fait de passer 10 heures par jour à naviguer sur le Net est nécessairement préférable à consacrer 10 heures à lire un livre ou à discuter avec des amies. De la même façon, notre recherche auprès des jeunes des écoles 
secondaires de l'Ontario nous a permis de constater que les filles sont moins nombreuses que les garçons à penser qu'il est plus agréable d'apprendre avec Internet qu'avec des livres. Elles sont aussi légèrement plus nombreuses que les garçons à considérer qu'il est plus efficace de faire des recherches avec des livres que sur Internet. Cette plus grande valorisation du livre chez les filles que chez les garçons peut être mise en parallèle avec la propension plus marquée chez les femmes que chez les hommes pour la pratique de la lecture (Séguin-Noël et Garon, 2000). Est-ce que la valorisation de la lecture et du livre chez les filles et leur usage moins intensif d'Internet constituent nécessairement des problèmes auxquels il faut apporter des solutions? Peut-être, mais en l'absence de données plus précises sur ce que les femmes et les hommes font en ligne et comment ces activités s'insèrent dans l'ensemble de leur vie quotidienne, il est difficile de conclure qu'un usage plus intensif est nécessairement positif.

\section{Les groupes de femmes francophones en situation minoritaire au Canada}

Notre enquête, réalisée en collaboration avec la Fédération nationale des femmes canadiennes-françaises, avait comme objectifs (1) de dresser un portrait de l'utilisation des TIC chez un échantillon d'une cinquantaine de groupes de femmes francophones en situation minoritaire au Canada, (2) d'évaluer leur connaissance et leur utilisation des programmes d'Industrie Canada et (3) d'élaborer des recommandations visant à accroître l'utilisation des TIC chez ces femmes.

L'échantillon était tiré d'une liste de 100 groupes de femmes affiliés à la FNFCF et de certains autres groupes qui s'occupent de la condition féminine en général plutôt que de secteurs spécifiques, tels la violence ou l'alphabétisation. Il visait à inclure des groupes représentatifs de la distribution des francophones par province. S'il y avait plusieurs groupes dans une communauté, un 
seul était retenu. Une personne désignée par le groupe était interviewée par téléphone en utilisant un questionnaire composé principalement de questions fermées. Le questionnaire avait déjà été envoyé à la personne afin de permettre une préparation des réponses, s'il y avait lieu.

À peu près la moitié des groupes avait un ordinateur et un peu plus avait accès à Internet, parfois grâce à l'accès qu'avaient certains membres du groupe à titre personnel. Les groupes estimaient que la moitié de leurs membres avaient accès à Internet, soit de chez elles, soit au travail, soit à partir d'un lieu public. On observe une forte association entre la région, la taille des groupes et l'accès aux ordinateurs et à Internet: dans l'Ouest et le Nord, les groupes sont plus grands et mieux équipés tandis que dans l'Est, ils sont plus petits et moins équipés. Dans la région de l'Ouest et du Nord, 87,5\% des groupes ont accès à Internet en tant que groupe à comparer à 36,8\% dans l'Est et à 33,3\% dans la région du Centre. En ce qui concerne la taille du groupe, 77,8\% des groupes comptant plus de 50 membres ont accès à Internet à comparer à 37,5\% des groupes ayant 50 membres ou moins. Les raisons de ces variations selon les régions restent à explorer.

Parmi les groupes sans accès, la moitié n'était pas intéressée à l'avoir, à cause d'absence de ressources (taille du groupe, financement, un local, compétence technique... ) en combinaison avec le fait que l'utilité des TIC n'était pas évidente pour le groupe. Les groupes sans accès mais intéressés à l'obtenir étaient généralement convaincus de l'utilité d'être branchés, tout en partageant les mêmes contraintes quant aux ressources (surtout absence d'un local et/ou de financement). Les usages qu'ils envisageaient rejoignaient ceux identifiés par les groupes déjà branchés, tout en étant moins nombreux.

La communication - surtout le courriel, la recherche de financement, la diffusion d'information (sur le groupe) — et la recherche informatisée sur le Web sont les formes d'utilisation d'Internet les plus souvent mentionnées tant par les groupes branchés que par ceux qui voudraient l'être. La principale différence entre ces deux catégories de groupes est que les branchés 
sont plus nombreux à dire utiliser ces différentes formes de communication que les aspirants branchés disent prévoir le faire. C'est comme si avec l'accès venait une meilleure sensibilité au potentiel qui, lui, amène à une utilisation plus diversifiée. Par contre, le commerce électronique et les conférences informatisées sont des activités très rarement pratiquées par les groupes; la participation à des groupes de discussion est plutôt exceptionnelle.

Certains obstacles à l'utilisation d'Internet sont identifiés tant par les branchés que les non branchés: coût de la mise à niveau des ordinateurs et des logiciels, manque de formation et contraintes budgétaires. À ceux-ci s'ajoutent, surtout pour les non utilisateurs, les coûts de l'équipement et les frais de branchement, la lenteur des connexions, un manque de sensibilité à l'utilité et les difficultés de l'adoption par l'ensemble des membres du groupe. D'autres obstacles qui sont surtout mentionnés par les utilisatrices touchent les problèmes techniques reliés à l'incompatibilité entre logiciels et à l'utilisation du français, le manque de contenu en français et un trop grand volume d'informations (qui sont d'ailleurs plus ou moins pertinentes). Il est à souligner que la communication en français avec leurs membres est une priorité pour les groupes, de même que l'accès à de l'information pertinente à leur réalité comme femmes francophones en milieu minoritaire. Trop souvent, au moins une de ces spécificités manque dans l'information à laquelle les groupes ont accès en utilisant le Web.

Les résultats de cette étude soulignent une ouverture de la part de la majorité des groupes de femmes à l'utilisation des TIC, mais d'importants écarts en ce qui concerne l'accès et l'utilisation actuels, notamment selon la région et la taille du groupe. D'autres obstacles ont également été identifiés par les représentantes de ces groupes de femmes en situation minoritaire, que ce soit en raison d'un contenu pertinent et en langue française, d'un manque de financement, d'un manque de formation ou d'un excédent d'informations dont le contenu est d'intérêt marginal. Afin d'encourager et de faciliter l'utilisation de ces TIC, il serait important de communiquer l'information sur les programmes de subvention et sur le potentiel qu'offrent les TIC dans un langage accessible et de plusieurs façons (ateliers, personnes ressources, 
documents sur papier et sur Internet), et d'offrir des possibilités d'essai gratuit pour une durée limitée.Ainsi, des mesures proactives sont essentielles si l'on veut réduire l'écart numérique qui reste très présent y compris parmi les groupes de femmes.

En conclusion de ces études, on peut affirmer que les facteurs responsables de l'écart numérique sont multiples et varient selon les groupes sociaux. Pour ce qui est de l'écart entre les femmes et les hommes, il semble qu'il s'agisse de facteurs principalement économiques. L'écart entre femmes francophones et anglophones, tout comme l'écart entre groupes de femmes de l'Est par rapport à ceux de l'Ouest et du Nord, par contre, fait appel à des facteurs additionnels, liés notamment aux ressources économiques, au manque de sensibilisation aux bénéfices potentiels des TIC, à l'absence d'une masse critique de personnes branchées et à la pénurie de contenu francophone pertinent sur le Web. Finalement, nous rejoignons le concert des voix actuelles qui constatent un besoin criant d'études plus précises et plus nuancées des différents usages d'Internet dans la vie quotidienne. On ne peut pas supposer que les technologies d'information et de communication, qui recouvrent une diversité d'applications (navigation sur le Web, courriel, forums de discussion, création de pages Web et de contenu) dans une diversité de situations (loisir, travail rémunéré, travail non-rémunéré), ont des effets sociaux uniformément positifs ou négatifs, ni que certaines catégories sociales sont totalement démunies et retardataires face aux bienfaits de la technologie.

\section{Bibliographie}

Castells, Manuel. 1996. The Rise of the Network Society, vol. 1, Cambridge, Mass. : Blackwell Publishers.

Denis, Ann et Michèle Ollivier. Sous presse. «How wired are Canadian women? The intersection of gender, class and language with the use of New Information Technologies» in A New Wave: Women and Research Across the Disciplines, Andrea Martinez and Meryn Stuart (eds.). Toronto, Sumach Press.

Denis, Ann et Michèle Ollivier. 2002. «Nouvelles technologies d'information et de communication: accès et usage chez les jeunes filles et garçons francophones en Ontario», Revue Francophonies d'Amérique, no. 12, automne : 37-49.

Duque, Nina. 1998. Recherche sur les impacts des nouvelles technologies d'information et de communication 
(NTIC) dans les groupes de femmes du Québec: difficultés et potentiel. Montréal, Relais-Femmes.

Fong, Eric, Barry Wellman, Melissa Kew and Rima Wilkes. 2001. «Correlates of the Digital Divide: Individual, Household and SpatialVariation", en ligne à http://www.chass.utoronto.ca/ wellman/ publications/index.html, consulté en septembre 2002.

Mohanty, Chandra Tapalde. 1997. «UnderWestern Eyes: Feminist Scholarship and Colonial Discourses», in Anne McClintock,Aamir Mufti and Ella Sohat, Dangerous Liaisons: Gender, Nation, and Postcolonial Perspectives, Minneapolis \& London, University of Minnesota Press: 255-277.

Ollivier, Michèle et Ann Denis. 2002. Les femmes francophones en situation minoritaire au Canada et les technologies d'information et de communication. Rapport préparé pour la Fédération nationale des femmes canadiennes françaises et Industrie Canada, mars.

Séguin-Noël, Rosalie et Rosaire Garon. 2000. Les pratiques culturelles des jeunes de 15 à 35 ans en 1999. Québec, Ministère de la Culture et des Communications, en ligne à http:// mcc.quebectel.qc.ca/Mcc/ClinStat.nsf/theme?OpenView. 